International Mathematical Forum, Vol. 8, 2013, no. 30, 1493 - 1501

HIKARI Ltd, www.m-hikari.com

http://dx.doi.org/10.12988/imf.2013.37146

\title{
Taylor Exactness, SVEP and Spectral Mapping Theorems
}

\author{
Muneo Chō \\ Department of Mathematics, Faculty of Science \\ Kanagawa University, Hiratsuka 259-1293, Japan \\ chiyom01@kanagawa-u.ac.jp \\ Robin Harte \\ School of Mathematics, Trinity college \\ Dublin 2, Ireland \\ hartere@gmail.com \\ Copyright (C) 2013 Muneo Chō and Robin Harte. This is an open access article dis- \\ tributed under the Creative Commons Attribution License, which permits unrestricted use, \\ distribution, and reproduction in any medium, provided the original work is properly cited.
}

Abstract. Spectral mapping theorems for "local spectra" derived from the holomorphic range and the single valued extension property are proved with the aid of "Taylor exactness".

Mathematics Subject Classification: 47A05, 47A11

Keywords: vector space, Taylor spectrum, spectral mapping theorem

\section{Introduction}

Most spectral mapping theorems are a combination of the remainder theorem for polynomials (the easy half), and the fundamental theorem of algebra. For example if linear operators $S$ and $T$ commute on a vector space $X$, in the sense that

$$
S T=T S
$$


then there is inclusion

$$
S^{-1}(0)+T^{-1}(0) \subseteq(S T)^{-1}(0)
$$

and inclusion

$$
(S T)(X) \subseteq S(X) \cap T(X),
$$

which is enough to give about half the spectral mapping theorem for both the point and the defect spectrum; for the failure of equality look [HHS] at

$$
S=T=\left(\begin{array}{ll}
0 & 1 \\
0 & 0
\end{array}\right) \text {. }
$$

For certain more sophisticated concepts of spectrum Vladimir Müller [Mü] has shown how we should also draw on the Euclidean algorithm [H1], [H3].

To see how this can be put to work we recall some of the infrastructure from the theory of the "Taylor spectrum": Whether or not they commute, the operator pair $S, T$ on $X$ induce a sequence of two operators $\delta_{0}, \delta_{1}$ from $X$ to $X \oplus X$ to $X$, let

$$
0 \longrightarrow X \stackrel{\delta_{0}}{\longrightarrow} X \oplus X \stackrel{\delta_{1}}{\longrightarrow} X \longrightarrow 0
$$

defined by setting

$$
\delta_{0}(x)=(-S x) \oplus(T x) ; \delta_{1}(x \oplus y)=T x+S y .
$$

Necessary and sufficient for the sequence (0.5) to satisfy the "chain complex condition"

$$
\delta_{1} \circ \delta_{0}=0
$$

is that the pair $(S, T)$ commute in the sense $(0.1)$.

If the sequence $\left(\delta_{1}, \delta_{0}\right)$ is exact, in the sense that

$$
\delta_{1}^{-1}(0) \subset \delta_{0}(X),
$$

we shall say that the pair $(S, T)$ is Taylor non singular. Now the "Taylor spectrum" $\sigma_{T}(S, T) \subset \mathbf{C}^{2}$ is the set of complex pairs $(\lambda, \mu)$ for which the operator pair $(S-\lambda I, T-\mu I)$ fails to be Taylor non singular [T].

1. Definition We shall call the pair $(S, T)$ of linear operators on the vector space $X$ left non singular if

$$
S^{-1}(0) \cap T^{-1}(0)=O \equiv\{0\},
$$

right non singular if

$$
S(X)+T(X)=X
$$

and middle non singular if

$$
(-S T)^{-1}(0) \subseteq\left(\begin{array}{c}
T \\
S
\end{array}\right) X
$$


Hence, $(S, T)$ is left non singular, right non singular and middle non singular if and only if $\left(\delta_{0}\right)^{-1}(0)=\{0\}, \delta_{0}(X \oplus X)=X$ and $\delta_{0}(X)=\delta_{1}^{-1}(0)$, respectvely.

Sufficient for all three conditions is that there exist $(U, V)$ for which

$$
\{U, V\} \subseteq \operatorname{comm}(S, T) \text { and } V S-T U=I,
$$

where $\operatorname{comm}(S, T)$ denotes the set of all operators which commute with $S$ and T. Sufficient for (1.4) is that

$$
\{S, T, U, V\} \text { is commutative and } V S-T U=I \text {, }
$$

in which case also for each $n \in \mathbf{N}$ there are $U_{n}, V_{n}$ for which

$$
\left\{U_{n}, V_{n}\right\} \subseteq \operatorname{comm}(S, T) \text { and } V_{n} S^{n}-T^{n} U_{n}=I .
$$

These definitions make sense for linear operators on vector spaces, in particular bounded operators on Banach spaces, whether or not they are commutative in the sense (0.1). For bounded operators $S, T$ of course the operators $U, V$ are understood to be bounded. The following ([H3] Theorem 4) was essentially shown by Manuel Gonzalez [G]:

2. Theorem Necessary and sufficient for the middle non singularity of $(S, T)$ are the following three conditions:

$$
\begin{gathered}
S^{-1}(0) \subseteq T S^{-1}(0) ; \\
T^{-1}(0) \subseteq S T^{-1}(0) ; \\
S(X) \cap T(X) \subseteq(S T)(T S-S T)^{-1}(0) .
\end{gathered}
$$

It then follows

$$
(S T)^{-1}(0)+(T S)^{-1}(0) \subseteq S^{-1}(0)+T^{-1}(0) .
$$

Spectral mapping theorems for "ascent" and "descent" follow:

3. Theorem If $(S, T)$ is a commuting pair and middle non singular then

$$
\begin{array}{r}
S^{-1}(0) \cap S(X)+T^{-1}(0) \cap T(X)=(S T)^{-1}(0) \cap(S T)(X) ; \\
(S T)^{-1}(0)+S T(X)=\left(S^{-1}(0)+S(X)\right) \cap\left(T^{-1}(0)+(T)(X)\right) .
\end{array}
$$

Proof of (3.1). Let $x \in S^{-1}(0) \cap(S)(X)$. Hence $S x=0$ and $x=S x_{1}$ for some $x_{1}$. Since $-S x+T 0=0$, by middle non singular there exists $x_{1}$ such that $x=T x_{2}$ and $0=S x_{2}$. Hence $-S x_{1}+T x_{2}=0$. By middle non singular there exists $z$ such that $x_{1}=T z$ and $x_{2}=S z$. Therefore, $x=T x_{2}=T S z$ and $x \in(S T)(X)$. Moreover, $S x=S T x_{2}=T S x_{2}=0$ and $x \in(S T)^{-1}(0)$.

Similarly, if $y \in T^{-1}(0) \cap T(X)$, it holds $y \in(S T)^{-1}(0) \cap(S T)(X)$. Conversely let $x \in(S T)^{-1}(0) \cap(S T)(X)$. Since $S(T x)=0$, by $-S(T x)+T 0=$ 0 there exists $y$ such that $T x=T y$ and $S y=0$. Since $T(x-y)=0$, by $-S 0+$ $T(x-y)=0$ there exists $z$ such that $T z=0$ and $x-y=S z$. Hence $x=y+S z$. Since $x=(S T) w$ for some $w, y=x-S z=S(T w-z) \in S(X)$. Therefore 
$y \in S^{-1}(0) \cap S(X)$. It is clear $S z \in T^{-1}(0)$. Since $S y=0$, by $-S y+T 0=0$ there exists $u$ such that $y=T u$ and $S u=0$. Hence $S z=x-y=T(S w-u)$ and $S z \in T^{-1}(0) \cap T(X)$. Therefore $x=y+S z \in S^{-1}(0) \cap S(X)+T^{-1}(0) \cap T(X)$.

Proof of (3.2). Let $x \in(S T)^{-1}(0)$ and $y \in(S T)(X)$. Hence $S T x=0$ and $y=S T z$. Since $S(T x)=0$, by $-S(T x)+T 0=0, T x=T w$ and $0=S w$ for some $w$. By $-S 0+T(x-w)=0,0=T u$ and $x-w=S u$ for some $u$. Hence $x=w+S u$ and $x+y=w+S(u+T z) \in S^{-1}(0)+S(X)$.

Next since $T(S x)=0$, by $-S 0+T(S x)=0$ there exists $w_{1}$ such that $0=T w_{1}$ and $S x=S w_{1}$. By $S\left(x-w_{1}\right)=0$, by $-S\left(x-w_{1}\right)+T 0=0$ there exists $u_{1}$ such that $x-w_{1}=T u_{1}$ and $0=S u_{1}$. Hence by $x=w_{1}+T u_{1}$ it holds $x+y=w_{1}+T\left(u_{1}+S z\right) \in T^{-1}(0)+T(X)$.

Conversely let $x \in\left(S^{-1}(0)+S(X)\right) \cap\left(T^{-1}(0)+T(X)\right)$. Hence $x=x_{1}+S x_{2}=$ $y_{1}+T y_{2}$ for some $x_{1}, x_{2}, y_{1}, y_{2}$ such that $S x_{1}=T y_{1}=0$. By (2.1) and (2.2) there exist $z, w$ such that $x_{1}=T z, y_{1}=S w$ and $S z=T w=0$. Hence

$$
x=T z+S x_{2}=S w+T y_{2} .
$$

By $-S\left(w-x_{2}\right)+T\left(z-y_{2}\right)=0, w-x_{2}=T u$ and $z-y_{2}=S u$ for some $u$. Since $x_{2}=w-T u$,

$$
x=T z+S x_{2}=(T z+S w)-S T u .
$$

Since $S T(T z+S w)=T^{2} S z+S^{2} T w=0, T z+S w \in(S T)^{-1}(0)$ and hence $x \in(S T)^{-1}(0)+(S T)(X)$.

4. Corollary Under the assumption of Theorem 3, then

$$
\begin{aligned}
& S^{-1}(0) \cap S(X)=O=T^{-1}(0) \cap T(X) \Longleftrightarrow(S T)^{-1}(0) \cap(T S)(X)=O ; \\
& S^{-1}(0)+S(X)=X=T^{-1}(0)+T(X) \Longleftrightarrow(S T)^{-1}(0)+(S T)(X)=X .
\end{aligned}
$$

Moreover it holds

$$
\begin{gathered}
\left(T\left(S^{-}(0)\right)+S(X)\right) \cap\left(S\left(T^{-1}(0)\right)+T(X)\right) \\
=S\left(T^{-1}(0)\right)+T\left(S^{-1}(0)\right)+(S T)(X) \cap(T S)(X) .
\end{gathered}
$$

(4.1) and (4.2) follows directly from (3.1) and (3.2), respectively.

Proof of (4.3). Let $x \in\left(T\left(S^{-}(0)\right)+S(X)\right) \cap\left(S\left(T^{-1}(0)\right)+T(X)\right)$. Then there exist $y_{1} \in S^{-1}(0), y_{2} \in T^{-1}(0), z_{1}, z_{2} \in X$ such that

$$
x=T y_{1}+S z_{1}=S y_{2}+T z_{2} .
$$

Hence $-S\left(z_{1}-y_{2}\right)+T\left(z_{2}-y_{1}\right)=0$. By middle non singular there exists $w \in X$ such that $z_{1}-y_{2}=T w$ and $z_{2}-y_{1}=S w$.

Therefore $x=T y_{1}+S y_{2}+S T w=S y_{2}+T y_{1}+T S w$ and

$$
x \in S\left(T^{-1}(0)\right)+T\left(S^{-1}(0)\right)+(S T)(X) \cap(T S)(X) .
$$


Conversely if $x \in S\left(T^{-1}(0)\right)+T\left(S^{-1}(0)\right)+(S T)(X) \cap(T S)(X)$ then there exist $y \in T^{-1}(0), z \in S^{-1}(0), w_{1}, w_{2} \in X$ such that

$$
x=S y+T z+S T w_{1}=S y+T z+T S w_{2} .
$$

Hence $x=T z+S\left(y+T w_{1}\right)=S y+T\left(z+S w_{2}\right)$ and $x \in\left(T\left(S^{-}(0)\right)+S(X)\right) \cap\left(S\left(T^{-1}(0)\right)+T(X)\right)$.

The conditions in (4.1) say that the operators $S, T$ and then $S T$ are of ascent $\leq 1$, and the conditions in (4.2) that they are of descent $\leq 1$. Theorem 3 therefore says that ascent $\leq 1$, and descent $\leq 1$, are "Müller regularities" [Mü]. If an operator $T^{k}$ has ascent, or descent, $\leq 1$, then the operator $T$ has ascent, or descent, $\leq k$. Thus if (1.6) holds then the implications (4.1) and (4.2) hold for all powers $S^{n}$ and $T^{n}$, and hence each of "finite ascent" and "finite descent" are Müller regularities.

To establish "local" analogues we need to replace ranges of operators by couer analytique and, on the way to that, couer algébrique:

5. Definition The couer algébrique of a linear operator $T: X \rightarrow X$ is

$$
T^{\varphi}(X)=\bigcup\{Y \subseteq X: Y=T(Y)\},
$$

the largest invariant subspace to which the restriction of the operator is onto.

Concretely it can be realized in terms of sequences $\xi=\left\{\xi_{n}\right\}_{n=0}^{\infty} \in X^{\infty}$, with the first infinite ordinal

$$
\infty=\mathbf{N}_{\bullet}=\mathbf{N} \cup\{0\}
$$

in the form

$$
T^{\varphi}(X)=\left\{\xi_{0}: \xi=\left\{\xi_{n}\right\}_{n=0}^{\infty} \in X^{\infty}, \xi_{n}=T\left(\xi_{n+1}\right)(\forall n \in \infty)\right\} .
$$

For the simplicity we denote $\xi=\left\{\xi_{n}\right\}_{n=0}^{\infty}$ by $\xi$. If we think of the sequences as belonging to a tensor product

$$
X^{\infty}=X \otimes \mathbf{C}^{\infty}
$$

then we can write

$$
T^{\varphi}(X)=\left\{\xi_{0} \in X: \xi \in(I \otimes 1-T \otimes v)^{-1}(0) \subseteq X^{\infty}\right\},
$$

where

$$
v:\left(\lambda_{0}, \lambda_{1}, \lambda_{2} \ldots\right) \mapsto\left(\lambda_{1}, \lambda_{2}, \lambda_{3} \ldots\right): \mathbf{C}^{\infty} \rightarrow \mathbf{C}^{\infty}
$$

is the backward unilateral shift; then also with

$$
u:\left(\lambda_{0}, \lambda_{1}, \lambda_{2} \ldots\right) \mapsto\left(0, \lambda_{0}, \lambda_{1} \ldots\right): \mathbf{C}^{\infty} \rightarrow \mathbf{C}^{\infty}
$$

the forward shift we find ([H6] Theorem 10)

$$
T^{-1}(0) \cap T^{\varphi}(X)=\left\{\xi_{0} \in X: \xi \in(I \otimes u-T \otimes 1)^{-1}(0) \subseteq X^{\infty}\right\} .
$$


Another characterization ([CHM] Theorem 2) is that $T^{\varphi}(X)=T^{\alpha}(X)$ whenever

$$
\operatorname{dim}(X) \leq|\alpha|
$$

the ordinal $\alpha$ has cardinal greater than the Hamel dimension of the linear space $X$. To extend Theorem 3 we must first extend Theorem 2 :

6. Theorem If $(S, T)$ is middle non singular then

$$
\begin{aligned}
& S^{-1}(0) \subseteq T^{\varphi} S^{-1}(0) \subseteq T^{\varphi}(X), \\
& T^{-1}(0) \subseteq S^{\varphi} T^{-1}(0) \subseteq S^{\varphi}(X) .
\end{aligned}
$$

Proofs of (6.1) and (6.2) are easy. Because let $x \in S^{-1}(0)$. Then since $S x=0$, by middle non singular there exists $x_{1}$ such that $x=T x_{1}$ and $S x_{1}=0$. Since $S x_{1}=0$, repeating this there exist $x_{n}$ such that $x_{n}=T x_{n+1}$ and $S x_{n}=0$. Hence $x \in T^{\varphi} S^{-1}(0)$. Similarly, (6.2) follows.

7. Theorem If there exist $U_{n}, V_{n}$ such that $\left\{S, T, U_{n}, V_{n}\right\}_{n=1}^{\infty}$ are commutative and $V_{1} S-U_{1} T=I, S V_{1} V_{1}=V_{1}, T U_{1} U_{1}=U_{1}, S V_{n+1} V_{1}=V_{n} V_{1}, T U_{n+1} U_{1}=$ $U_{n} U_{1}$ then

$$
S^{\varphi}(X) \cap T^{\varphi}(X) \subset(S T)^{\varphi}(X)
$$

Proof. Let $w \in S^{\varphi}(X) \cap T^{\varphi}(X)$. Then there exist sequences $\left\{x_{n}\right\},\left\{y_{n}\right\}$ such that

$$
w=S x_{0}=T y_{0}, \quad x_{n}=S x_{n+1}, \quad y_{n}=T y_{n+1}, \quad \forall n \in \infty .
$$

Let $z_{0}=V_{1} y_{0}-U_{1} x_{0}$ and $z_{n}=V_{n} V_{1} y_{n}-U_{n} U_{1} x_{n}$ for $n \geq 1$. Then $(S T) z_{0}=w$ is clear,

$$
(S T) z_{1}=S V_{1} V_{1} T y_{1}-T U_{1} U_{1} S x_{1}=V_{1} y_{0}-U_{1} x_{0}=z_{0}
$$

and

$$
(S T) z_{n+1}=\left(S V_{n+1} V_{1}\right) T y_{n+1}-\left(T U_{n+1} U_{1}\right) S x_{n+1}=V_{n} V_{1} y_{n}-U_{n} U_{1} x_{n}=z_{n} .
$$

Hence $w \in(S T)^{\varphi}(X)$.

The analogue of Theorem 3 with couer algébrique in place of ranges now follows, arguing as for Theorem 3.

Local spectral theory begins with the couer analytique or holomorphic range:

8. Definition The holomorphic range of $T \in B(X)$ is the set of $x \in X$ for which there is $f \in \operatorname{Holo}(0, X)$ for which

$$
(T-z I) f(z) \equiv x
$$

The holomorphic kernel is the set of $x \in X$ for which there is $g \in \operatorname{Holo}(0, X)$ for which

$$
x=g(0) \text { and }(T-z I) g(z) \equiv 0 .
$$


If we write

$$
k_{\infty}(X)=\left\{\xi \in X^{\infty}: \sup _{n}\left\|\xi_{n}\right\|^{1 / n}<\infty\right\},
$$

then we can realize the holomorphic range as the space

$$
T^{\omega}(X)=\left\{x \in X: \exists \xi \in k_{\infty}(X): \xi_{0}=x, \xi_{n}=T\left(\xi_{n+1}\right)(\forall n \in \infty)\right\} \subseteq T^{\varphi}(X)
$$

and the holomorphic kernel ([H6] Theorem 9) as the intersection

$$
T^{-1}(0) \cap T^{\omega}(X) \text {. }
$$

In contrast to the set $\Xi(X)$ of $[\mathrm{H} 6], k_{\infty}(X)$ here is a linear subspace of the sequence space $X^{\infty}$, and indeed a Banach space in its own right:

$$
c_{0}(X) \subseteq k_{\infty}(X) \subseteq \ell_{\infty}(X) .
$$

For an alternative version of the couer algébrique we could replace the holomorphic functions of Definition 8 by formal power series. If we declare $T \in B(X)$ to be holomorphically one one whenever

$$
T^{-1}(0) \cap T^{\omega}(X)=\{0\},
$$

then ([H6] Theorem 10)

$$
T \text { holomorphically one one } \Longleftrightarrow T \text { has SVEP at } 0 \in \mathbf{C} \text {. }
$$

Note [H7] the cosmetic misprint in the statement in [H6].

Theorem 2 and Theorem 3 also (cf [Mü] Lemma 14.1) hold with holomorphic ranges in place of ranges: the analogue of Theorem 2 follows easily from Theorem 6. When $S$ and $T$ commute then the inclusions (2.1) and (2.2) become equalities and the open mapping theorem comes into play:

9. Theorem If there exist $U_{n}, V_{n}$ such that $\left\{S, T, U_{n}, V_{n}\right\}_{n=1}^{\infty}$ are commutative and $V_{1} S-U_{1} T=I, S V_{1} V_{1}=V_{1}, T U_{1} U_{1}=U_{1}, S V_{n+1} V_{1}=V_{n} V_{1}, T U_{n+1} U_{1}=$ $U_{n} U_{1}$ then

$$
\left(S^{-1}(0) \cap S^{\omega}(X)\right)+\left(T^{-1}(0) \cap T^{\omega}(X)\right)=(S T)^{-1}(0) \cap(S T)^{\omega}(X)
$$

and

$$
(S T)^{-1}(0)+(S T)^{\omega}(X)=\left(S^{-1}(0)+S^{\omega}(X)\right) \cap\left(T^{-1}(0)+T^{\omega}(X)\right) .
$$

Proof. Theorem 2 with holomorphic ranges in place of ranges says

$$
S^{-1}(0) \subseteq T^{\omega}(X) ; T^{-1}(0) \subseteq S^{\omega}(X) ; S^{\omega}(X) \cap T^{\omega}(X) \subseteq(S T)^{\omega}(X),
$$

together with of course (2.4); now go again to the argument of Theorem 3. Therefore (9.1) and (9.2) hold.

The spectral mapping theorem for the SVEP spectrum is given by Müller ([Mü] Theorem 14.6, Corollary 14.7), Aiena ([A] Theorem 2.39; [AMG] Theorem 5), Laursen and Neumann ([LM] Theorem 3.36) and Colojoara and Foias ([CF] Theorems 1.5, 1.6). Theorem 9 tells us, proving Theorem 11 of [H6], 
that operators which are holomorphically one one constitute a Müller regularity. For a corresponding notion of holomorphically bounded below we might consider the operators which "have Property $(\beta)$ at $0 \in \mathbf{C}^{\prime}$, or alternatively [H7] operators $T \in B(X)$ whose enlargements $\mathbf{Q}(T) \in B\left(\ell_{\infty}(X) / c_{0}(X)\right)$ are holomorphically one one. A third alternative would be operators $T \in B(X)$ for which, in the notation of (5.6), there is $k>0$ for which, for arbitrary $\xi \in k_{\infty}(X)$

$$
\left\|\xi_{0}\right\| \leq k\|(I \otimes u-T \otimes 1)(\xi)\| .
$$

Theorem 9 also tells us that local spectrum comes from a Müller regularity, where it is traditional to write, for $x \in X$,

$$
\lambda \notin \sigma_{T}(x) \Longleftrightarrow x \in(T-\lambda I)^{\omega}(X) .
$$

\section{REFERENCES}

[1] [A]P. Aiena, Multipliers, local spectral theory and Fredholm theory, Kluwer 2004.

[2] [AMN]P. Aiena, T.L. Miller and M.M. Neumann, On a localised single valued extension property, Proc. R.I.A. 104A (2004) 17-34

[3] [CHM]M. Chō, R.E. Harte and V. Müller, Transfinite ranges and the local spectrum, Jour. Math. Anal. Appl. (to appear).

[4] [CF]I. Colojoara and C. Foias, Theory of generalized spectral operators, Gordon and Breach 1968.

[5] [F]J.K. Finch, The single valued extension property on a Banach space, Pacific Jour. Math. 58 (1958) 61-69.

[6] [G]M. Gonzalez, Null spaces and ranges of polynomials of operators, Publ. Mat. Univ. Barcelona 32 (1988) 167-1270.

[7] [H1]R.E. Harte, Cayley-Hamilton for eigenvalues, Bull. Irish Math. Soc. 22 (1989) 6668.

[8] [H2]R.E. Harte, Invertibility and singularity (Dekker New York 1988).

[9] [H3]R.E. Harte, Taylor exactness and Kato's jump, Proc. Math. Soc. 119 (1993) 793801.

[10] [H4]R.E. Harte, On Kato non-singularity, Studia Math. 117 (1996) 107-114.

[11] [H5]R.E. Harte, Variations on a theme of Kaplansky, Filomat (Nis) 16 (2002) 19-30.

[12] [H6]R.E. Harte, On local spectral theory, Operator Theory: Advances and Applications 187 (2008) 175-183.

[13] [H7]R.E. Harte, On local spectral theory II, Funct. Anal. Approx. Comp. 2 (2010) 67-71.

[14] [HHS]R.E. Harte, C. Hernandez and C.M. Stack, Exactness and the Jordan form, Funct. Anal. Approx. Comp. 3 (2011), 1-7.

[15] [K]J.J. Koliha, Isolated spectral points, Proc. Amer. Math. Soc. 124 (1996) 3417-3424.

[16] [LN]K.B. Laursen and M.M. Neumann, Introduction to local spectral theory, Clarendon Press 2000.

[17] [Mb1]M. Mbekhta, Généralisation de la décomposition de Kato aux opérateurs paranormeaux et spectraux, Glasgow Math. Jour. 29(1987) 159-175.

[18] [Mb2]M. Mbekhta, Sur la théorie spectrale locale et limite des nilpotents, Proc. Amer. Math. Soc. 110 (1990) 621-631.

[19] [Mü]V. Müller, Spectral theory of linear operators, Birkhäuser Boston 2007. 
[20] [S]P. Saphar, Contribution a l'étude des applications linéares dans les espaces de Banach, Bull. Soc. Math. France 92 (1964) 363-384.

[21] [Sc]C. Schmoeger, On isolated points of the spectrum of a bounded linear operator, Proc. Amer. Math. Soc. 117 (1993) 715-719.

[22] [T]J.L. Taylor, A joint spectrum for several commuting operators, J. Functional Anal. 6 (1970) 172-191.

[23] [V]P. Vroba, On local spectral properties of operators in Banach spaces, Czech. Math. Jour. 23 (1973) 483-492.

Received: July 21, 2013 